\title{
UNUSUAL TIME DEPENDENCE OF MAGNETIZATION RELAXATION IN MBE GROWN EPILAYER OF CdMnTe*
}

\author{
A.M. Witowski \\ Institute of Experimental Physics, Warsaw University \\ Hoża 69, 00-681 Warszawa, Poland \\ H.-P. MOLL, P. WYDER
}

High Magnetic Field Laboratory, Max-Planck-Institut für Festkörperforschung Centre National de la Recherche Scientifique Grenoble, France

\author{
G. Karczewsin, T. Wojtowicz and J. Kossut \\ Institute of Physics, Polish Academy of Sciences \\ Al. Lotników 32/46, 02-668 Warszawa, Poland
}

The temporal behavior of the magnetization due to the spin-lattice relaxation for bulk and MBE grown epilayer of CdMnTe is compared. The time dependence of the epilayer magnetization changes $(\mathrm{d} M / \mathrm{d} t)$ is nonmonotonic. After the main part, a second structure appears. It can be explained only by the assumption of energy storage and a delayed additional rise of specimen temperature. The nature of the storage is unknown.

PACS numbers: $76.30 . \mathrm{Fc}, 68.60 . \mathrm{Wm}$

Our previous results of magnetization relaxation measurements for diluted magnetic semiconductors [1] show that the relaxation below an external magnetic field of $10 \mathrm{~T}$, for CdMnTe with Mn content below $2 \%$, is determined by the transfer of polarization to unknown defects or impurities [2]. The data for CdMnS [3] pointed out the importance of the growth process on this part of the relaxation. To check this effect we investigated materials grown by totally different methods, namely modified Bridgman and MBE. Both samples have an Mn content of about $10 \%$. The epilayer (Mn content $9.5 \%$ and In doping of the order of $10^{19} \mathrm{~cm}^{-3}$ ) was grown on a GaAs substrate with a thin ZnCdTe buffer (sample \#03074 CMT:In). The bulk material was undoped. It should be pointed out that for such a high

*This work is partially supported by the grant \# 224109203 of the State Committee for Scientific Research (Republic of Poland). 
concentration of magnetic impurities the spin-lattice relaxation responsible for the magnetization relaxation goes through spin clusters and is due to the modulation of the exchange interaction [2,4]. In this case a comparison of two results can give some information about the formation of the clusters. We should stress that it also gives an experimental check of the sensitivity of the used measuring method.

We measured the magnetization relaxation using the nonresonant method described in [5]. After a short laser pulse that warms the lattice, the spin system warms up to the new temperature by means of spin-lattice relaxation [4], changing the sample magnetization (given by the spins' alignment). These changes induced a voltage in the pick-up coil. For the bulk crystals the amplitude of the induced voltage after a sharp rise at the beginning decays exponentially (spin-lattice relaxation time) $[4,5]$. Sometimes the decay is described by the sum of a few exponential functions, but is always monotonic. The typical temporal behavior of the induced voltage for a bulk CdMnTe sample is depicted in Fig. 1. The decay of the signal is very close to the coil limit (almost symmetric peak). It can be described by an exponential curve $(V(t) \sim \exp (-t / \tau))$ with the relaxation time $\tau_{\mathrm{b}}$ independent of the magnetic field (see Fig. 2).

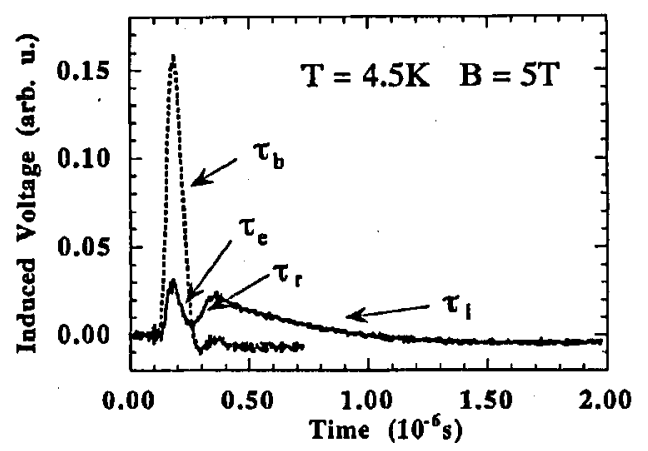

Fig. 1. Typical temporal behavior of the voltage induced in the coil for CdMnTe bulk sample with Mn content $10 \%$ (dashed line) and an MBE grown CdMnTe $10 \%$ epilayer (solid line). The sample temperatures were $4.5 \mathrm{~K}$ and the external magnetic field was $5 \mathrm{~T} . \tau_{\mathrm{b}}$ denotes the part of the data for the bulk sample to which an exponential function with a relaxation time $\tau_{\mathrm{b}}$ was fitted. $\tau_{\mathrm{e}}, \tau_{\mathrm{r}}$, and $n$ denote parts to which exponential functions with characteristic times $\tau_{e}, \tau_{r}$, and $\eta$ were fitted.

The MBE grown sample was placed in the same coil, with the layer parallel to the coil axis. In this case the temporal behavior of the induced voltage is nonmonotonic (Fig. 1). After a first part which is comparable with the signal for bulk sample, a second structure is observed. Its rise time $\tau_{\mathbf{r}}$ is longer than in the first peak and the decay is much longer (Fig. 2). Such temporal behavior (two peaks) cannot be explained by just a sum of exponential decays. It suggests that there is an additional energy reservoir in the system, which after a heating pulse releases energy with a certain delay. Because the relaxation of the spin system is much faster than the times involved for the structure, we observed the temporal 


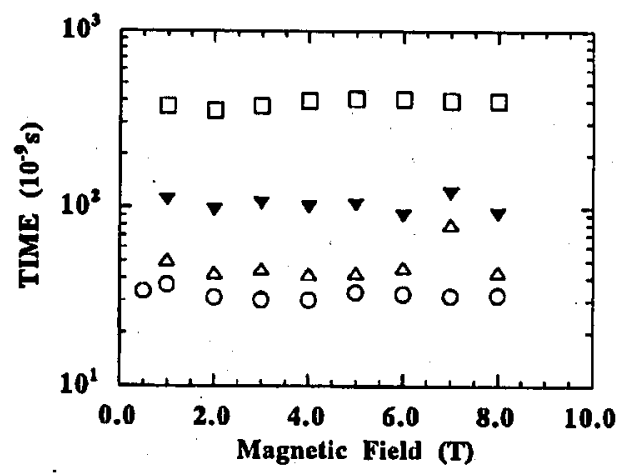

Fig. 2. The magnetic field dependence of the characteristic times $\tau_{\mathrm{b}}, \tau_{\mathrm{e}}, \tau_{\mathrm{r}}$, and $n$ observed in experiments (see Fig. 1). The decay time for the bulk sample $\tau_{\mathrm{b}}$ is represented by circles. The first $\tau_{\mathrm{e}}$ and the second $\eta$ decay times for the epilayer are shown by open triangles and open squares, respectively. Full triangles represent the rise time $\tau_{\mathrm{r}}$ of the second structure for the epilayer.

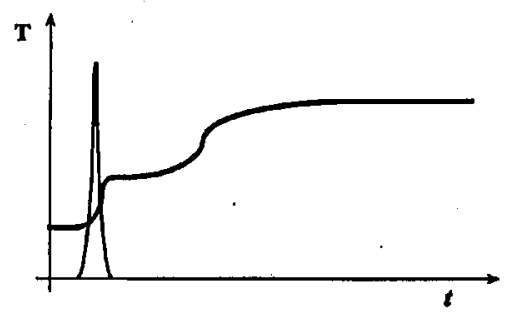

Fig. 3. Schematic representation of the temporal behavior of the epilayer lattice temperature deduced from the magnetization changes, the time derivative of which leads to the voltage induced in the coil and presented in Fig. 1. The light line presents the laser pulse.

behavior of the lattice temperature. Estimated temperature changes of the lattice should be like those presented in Fig. 3. The observed decay time characterizes the energy transfer. The origin of this additional reservoir of energy is unknown. It can be connected with defects and/or impurities in the substrate. Another possibility is the energy accumulation in the buffer layer or indium impurities. To answer which of these possibilities is the dominant one, more experiments carried out for different epilayers are needed.

\section{Acknowledgments}

We would like to thank M. Grynberg, M. Potemski and Ch. Kutter for valuable discussions. The authors are grateful to M.L. Sadowski for a critical reading of the manuscript. One of us (A.M.W.) would like to thank Max-Planck Gesellschaft for financial support during the stay in HMFL Grenoble. 


\section{References}

[1] For review see e.g. Diluted Magnetic Semiconductors, Eds. J.K. Furdyna, J. Kossut, in: Semimagnetic and Semimetals, Vol. 25, Academic, New York 1988; W.J.M. de Jonge, H.J.M. Swagten, J. Magn. Magn. Mater. 100, 322 (1991).

[2] T. Strutz, A.M. Witowski, P. Wyder, Phys. Rev. Lett. 68, 3912 (1992).

[3] A.M. Witowski, Ch. Kutter, W. Mac, P. Wyder, Acta Phys. Pol. A 84, 798 (1993).

[4] For review see e.g. K.J. Standley, R.A. Vaughan, Electron Spin Relaxation Phenomena in Solids, Hilger, London 1969; K.W.H. Stevens, Rep. Prog. Phys. 30, 189 (1967).

[5] T. Strutz, A.M. Witowski, P. Wyder, Rev. Sci. Instrum. 64, 1853 (1993). 